\title{
Efficacy of Acotiamide on Postprandial Distress Syndrome and Epigastric Pain Syndrome Depending on the Estimated Gastric Acid Secretion Level
}

\author{
Toshiaki Suzuki, ${ }^{1}$ Reina Ohba, ${ }^{1}$ Ei Kataoka, ${ }^{2}$ Yui Kudo, ${ }^{3}$ Akira Zeniya, ${ }^{4}$ Daisuke Segawa, ${ }^{5}$ Keisuke Oikawa, \\ Masaru Odashima, ${ }^{7}$ Taiji Saga, ${ }^{8}$ Tomoyuki Kuramitsu, ${ }^{9}$ Hideaki Sasahara, ${ }^{10}$ Kazuo Yoneyama, ${ }^{11}$ Takashi Tomita, ${ }^{12}$ \\ Yosuke Shimodaira, ${ }^{1}$ and Katsunori lijima ${ }^{1 *}$ \\ ${ }^{1}$ Department of Gastroenterology, Akita University Graduate School of Medicine, Akita, Japan; ${ }^{2}$ Kataoka Internal Medicine Clinic, Akita, \\ Japan; ${ }^{3}$ Kudo Gastroenterology Clinic, Akita, Japan; ${ }^{4}$ Zeniya Clinic, Akita, Japan; ${ }^{5}$ Segawa Clinic, Noshiro, Japan; ${ }^{6}$ Oikawa Clinic, Akita, Japan; \\ ${ }^{7}$ Odashima Masaru Clinic, Yokote, Japan; ${ }^{8}$ Matsugasaki Clinic, Akita, Japan; ${ }^{9}$ Kuramitsu Clinic, Akita, Japan; ${ }^{10}$ Sasahara Internal Medicine Clinic, \\ Akita, Japan; ${ }^{11}$ Yoneyama Gastroenterology Clinic, Akita, Japan; and ${ }^{12}$ Tomita Gastroenterology Clinic, Akita, Japan
}

\section{Background/Aims}

Gastric acid secretion is suspected to be a pivotal contributor to the pathogenesis of functional dyspepsia. The present study investigates the potential association of the gastric acid secretion estimated by measuring serum pepsinogen with therapeutic responsiveness to the prokinetic drug acotiamide.

\section{Methods}

Dyspeptic patients consulting participating clinics from October 2017 to March 2019 were prospectively enrolled in the study. The dyspeptic symptoms were classified into postprandial distress syndrome (PDS) and epigastric pain syndrome (EPS). Gastric acid secretion levels were estimated by the Helicobacter pylori infection status and serum pepsinogen using established criteria and classified into hypo-, normo-, and hyper-secretion. Each patient was then administered $100 \mathrm{mg}$ acotiamide thrice daily for 4 weeks, and the response rate to the treatment was evaluated using the overall treatment efficacy scale.

\section{Results}

Of the 86 enrolled patients, $56(65.1 \%)$ and 26 (30.2\%) were classified into PDS and EPS, respectively. The estimated gastric acid secretion was not significantly different between PDS and EPS. The response rates were $66.0 \%$ for PDS and $73.1 \%$ for EPS, showing no significant difference. While the response rates were stable, ranging from $61.0 \%$ to $75.0 \%$ regardless of the estimated gastric acid secretion level among subjects with PDF, the rates were significantly lower in hyper-secretors than in non-hyper-secretors among subjects with EPS $(42.0 \%$ vs $83.0 \%, P=0.046)$.

\section{Conclusion}

Although acotiamide is effective for treating EPS as well as PDS overall, the efficacy is somewhat limited in EPS with gastric acid hypersecretion, with gastric acid suppressants, such as proton pump inhibitors, being more suitable.

\section{(J Neurogastroenterol Motil 2022;28:53-61)}

Key Words

Acotiamide; Epigastric pain syndrome; Gastric acid secretion; Serum pepsinogen; Postprandial distress syndrome

Received: August 21, 2020 Revised: October 14, 2020 Accepted: October 24, 2020

(.) This is an Open Access article distributed under the terms of the Creative Commons Attribution Non-Commercial License (http://creativecommons. org/licenses/by-nc/4.0) which permits unrestricted non-commercial use, distribution, and reproduction in any medium, provided the original work is properly cited.

*Correspondence: Katsunori lijima, MD, PhD

Department of Gastroenterology, Akita University Graduate School of Medicine, 1-1-1 Hondo, Akita 010-8543, Akita, Japan Tel: +81-18-884-6573, Fax: +81-18-801-7501, E-mail: kiijima@med.akita-u.ac.jp 


\section{Introduction}

Functional dyspepsia (FD) is defined as a condition chronically presenting with upper abdominal symptoms in the absence of any organic disease that is likely to explain the symptoms, and a wide variety of factors is assumed to be involved in its pathophysiology. ${ }^{1}$ Based on the Rome $\mathrm{IV}^{2}$ FD is sub-classified into 2 major subtypes: postprandial distress syndrome (PDS) and epigastric pain syndrome (EPS). PDS is characterized by postprandial fullness and early satiety induced by ingestion of a meal, while EPS is characterized by epigastric pain and burning that occur during fasting. However, while a few studies have successfully demonstrated differences in the pathophysiology between the 2 subtypes of $\mathrm{FD}$, others have failed to detect any marked differences. ${ }^{5-7}$ At present, gastric acid suppression with a proton pump inhibitor (PPI) and improvement of gut motility with prokinetic drugs are the 2 main treatment options for FD. ${ }^{1}$ Assuming that the symptom pattern (PDS or EPS) may aid in determining the most appropriate firstline therapy, it is proposed that patients with PDS be treated with prokinetic drugs, while those with EPS are considered more likely to respond to a PPI. ${ }^{8-10}$ However, the concept of treatment choice for FD depending on the symptom pattern is not yet validated. ${ }^{11}$

Acotiamide, a new prokinetic drug that has been shown to improve gastric motility and gastric emptying, has been available in Japan for the treatment of FD since 2013. The phase III trial of the drug proved its efficacy for the treatment of meal-related symptoms, so it has been used mainly for PDS-type FD. ${ }^{12}$ While a recent retrospective study reported the efficacy of acotiamide for not only PDS but also EPS, ${ }^{13}$ this finding needs to be confirmed in a prospective study. In addition, the identification of clinical parameters to predict the responsiveness of the drug is of clinical significance, but few studies have addressed this issue. ${ }^{13,14}$

Gastric acid is one of the most important pathogenic factors influencing the development of FD, a notion supported by the fact that acid suppression with PPI has some effect on relieving FD symptoms and is often applied to such patients as first-line therapy. ${ }^{1,2}$ However, the involvement of gastric acid in the manifestation of FD symptoms is considered to be complicated. For example, strong acid externally administered to the stomach or the duodenal bulb induced dyspeptic symptoms in healthy volunteers and patients with FD ${ }^{15,16}$ However, hypochlorhydric patients is more likely to be associated dysmotility symptoms in patients with $\mathrm{FD}$, as we previously reported. ${ }^{17}$ Furthermore, although subjects' specific gastric acid secretion levels may be able to modulate the responsive- ness of drugs used for FD treatment, no study has yet investigated the efficacy of such drugs depending on the gastric acid secretion level.

Pepsinogen I is secreted exclusively by the fundic glands, and pepsinogen II is secreted by the fundic glands, pyloric glands, and proximal duodenal mucosa. ${ }^{18}$ Many previous studies have shown that the serum pepsinogen (sPG) I level and/or I/II ratio reflect the gastric acid secretion level. ${ }^{19,20}$ Based on the accumulated data on gastric acid secretion and sPG from a total of 627 Japanese patients, we previously established reliable cutoff $\mathrm{sPG}$ values to predict the gastric acid secretion status. ${ }^{21}$ Furthermore, using these criteria, we showed that estimating the gastric acid secretion levels by simply measuring the sPG is useful for predicting the risks of various acidrelated diseases of the upper gastrointestinal tract. ${ }^{22}$

We conducted this multi-clinic singe-arm prospective study as a pilot study, exploring the potential associations of the individual gastric acid secretion level estimated by measuring the $\mathrm{PGG}$ with the type of FD or efficacy of acotiamide treatment in patients with FD.

\section{Materials and Methods}

\section{Patients}

Patients $\geq 20$ years old who consulted participating clinics from October 2017 to March 2019 with complaints of chronic symptoms centered in the upper abdomen for more than 1 month in the absence of organic diseases likely to explain the symptoms were asked to answer a validated questionnaire using a global overall symptom (GOS) score. ${ }^{23,24}$ In the questionnaire, patients rated 8 overall abdominal symptoms experienced in the previous week using a 7-point Likert scale ranging from a score of 1 for "not at all bothered" to 7 for "unbearably bothered." The patients were then considered eligible and prospectively enrolled in the study if they had one or more specific upper gastrointestinal symptoms, such as postprandial distress (question 1), epigastric pain (question 4), early satiety and/or fullness (question 5), and epigastric burning (question 7) with at least moderate severity (score $\geq 4$ ). The participating institutions were the following 11 clinics located in Akita prefecture, Japan: Kataoka Internal Medicine Clinic, Kudo Gastroenterology Clinic, Zeniya Clinic, Segawa Clinic, Oikawa Clinic, Odashima Masaru Clinic, Matsugasaki Clinic, Kuramitsu Clinic, Sasahara Internal Medicine Clinic, Yoneyama Gastroenterology Clinic, and Tomita Gastroenterology Clinic.

The exclusion criteria were as follows: those who had taken acid inhibitory drugs or prokinetic drugs within the past month; those 
taking non-steroidal anti-inflammatory drugs or aspirin; those with predominant symptoms suggestive of gastroesophageal reflux diseases or irritable bowel syndrome, although concurrence with these symptoms was permitted; those with histories of operations for upper gastrointestinal tract; those who had received Helicobacter pylori eradication therapy within the past 6 months; and those with suspected psychological disorders or uncontrolled diabetes mellitus.

\section{Study Protocol}

The enrolled patients were interviewed regarding their body weight, height, smoking status, and history of $H$. pylori eradication therapy and were classified into 3 groups based on the predominant type of dyspeptic symptoms (PDS, EPS, and overlapping). The subgrouping of the symptoms was made based on each patient's chief complaint, regardless of the reported GOS scoring. Next, a blood sample was collected from each patient under fasting conditions to measure the serum $H$. pylori antibody, sPG I, and sPG II levels.

Each patient was then administered a $100 \mathrm{mg}$ tablet of acotiamide thrice a day for 4 weeks, and at the completion of the treatment, they revisited the clinic, answered the questionnaire concerning the GOS score, and reported on the efficacy of the treatment using an overall treatment efficacy (OTE) questionnaire. ${ }^{12,25}$ In the questionnaire, the patients made a global assessment of the change in their dyspeptic symptoms compared with the pretreatment baseline period using a 7-point Likert scale from "extremely improved" to "improved," "slightly improved," "not changed," "slightly aggravated," "aggravated," and "extremely aggravated." In the subsequent analysis, patients who reported "extremely improved" or "improved" on the OTE questionnaire were defined as responders, while other patients were defined as non-responders.

The study protocol was approved by the Akita University School of Medicine Ethics Committee (1779). All subjects provided their written informed consent prior to enrollment. The study was registered at the University Hospital Medical Information (UMIN000036265).

\section{Estimation of gastric acid secretion by Helicobacter py- lori infection and serum pepsinogen}

The $H$. pylori infection status was determined by the titers of serum IgG antibodies against $H$. pylori using an enzyme immunoassay kit (E Plate "Eiken" H. pylori antibody; Eiken Chemical Co. Ltd, Tokyo, Japan). The sPG I and sPG II levels were measured by a chemiluminescent enzyme immunoassay using commercial kits (Lumipulse PG I and II; Fujirebio Inc, Tokyo, Japan).
We previously demonstrated that the sPG I value correlated well with gastric acid secretion in $H$. pylori-negative subjects, while the sPG I/II ratio correlated well in $H$. pylori-positive subjects. ${ }^{19}$ We therefore defined gastric acid hypo-secretion as sPG I $<35 \mathrm{ng} /$ $\mathrm{mL}$ in $H$. pylori-negative subjects (area under the curve [AUC]: 0.80 ) and an I/ II ratio < 2.7 in $H$. pylori-positive subjects (AUC: $0.87){ }^{21}$ Similarly, we defined gastric acid hyper-secretion as sPG I values $>50 \mathrm{ng} / \mathrm{mL}$ in $H$. pylori-negative subjects (AUC: 0.79 ) and as an $\mathrm{I} / \mathrm{II}$ ratio $>3.3$ in $H$. pylori-positive subjects (AUC: $0.87) .^{21}$ All other subjects were defined as having gastric acid normo-secretion. In addition, since sPG I values were more wellcorrelated with gastric acid secretion than I/II ratios in $H$. pylorieradicated subjects, ${ }^{26}$ the same criteria as in $H$. pylori-negative subjects was applied to the eradicated subjects.

\section{Statistical Methods}

Results were expressed as the mean (SD) for continuous variables, and ratios (percentages) for categorical variables. Comparisons between PDS and EPS, or responders and non-responders were performed either by an unpaired $t$ test for continuous variables or by a chi-square test for categorical variables. Response rates were calculated as percentages of responders among those who completed a 4-week treatment protocol and were compared using the chi-square test among subgroups of estimated gastric acid secretion. In addition, using the GOS score before and after treatment in each patient, a PDS symptom severity score was calculated by summing postprandial fullness (question 1) and early satiety (question 5), and an EPS symptom severity score was calculated by summing epigastric pain (question 4) and epigastric burning (question 7). Those symptom severity scores were expressed as the median (interquartile range), with the values compared between patients with PDS and EPS by the Mann-Whitney $U$ test and before and after treatment by Wilcoxon rank sum test. All analyses were performed using the JMP Pro 13 software program (SAS Institute Inc, Cary, NC, USA), and a $P$-value $<0.05$ was considered to indicate a statistically significant difference.

Regarding the sample size, since there have been no previous studies investigating the efficacy of prokinetic drugs by the gastric acid secretion level, we set a sample size of 200 for this pilot study considering the feasibility of subject enrollment in our area.

\section{Results}

Initially, 88 patients with $\mathrm{FD}$ symptoms consulting the participating clinics agreed to participate in this study, but 2 were deemed 
ineligible for the study (PPI had already been administered in 1 case, and the intensity of the symptoms was insufficient for inclusion according to the GOS questionnaire in the other case). Consequently, a total of 86 patients were enrolled in this study and subjected to the initial analysis for comparing the clinical parameters between PDS and EPS. Treatment with acotiamide was commenced in all of these patients, including 2 who were lost to follow-up and 1 who was administered a minor tranquilizer at another hospital. Ultimately, 83 patients completed the 4-week treatment course and were subjected to the analysis of the efficacy of acotiamide. No one had taken acotiamide previously.

Among the 86 enrolled patients, the majority $(72.1 \%)$ were female with a mean age of 54.8 (15.2) years old. The period of illness before consulting the clinic was 1-3 months in 60.5\%, 3-6 months in $16.3 \%$, and $>6$ months in $23.2 \%$. Concerning $H$. pylori infection status, the vast majority $(87.2 \%)$ were judged to be negative (naïve-negative in $75.6 \%$ and eradicated in $15.1 \%$ ), while $12.8 \%$ were $H$. pylori-positive. In terms of the estimated gastric acid secretion level, 44 (51.2\%), 27 (31.4\%), and 15 (17.4\%) were classified as hypo-secretors, normo-secretors, and hyper-secretors, respectively (Table 1).

Based on the predominant chief complaint of dyspeptic symptoms, $56(65.1 \%)$ and $26(30.2 \%)$ were defined as having PDS and EPS, respectively, while the remaining 4 (4.6\%) were overlapping. It should be noted that, since subgrouping of the symptoms was made based on each patient's chief complaint and not by the symptom score, although the PDS symptom severity score was significantly higher than the EPS score in patients with PDS (7.0 [2.0] vs 5.0 [2.0], $P<0.01$ ), both scores were similarly high in patients with EPS (PDS score: 6.0 [3.0] vs EPS score 6.0 [2.0]) (Fig. 1).

On comparing the clinical parameters between PDS and EPS, there was no significant difference in any studied parameters between the 2 groups (Table 1). Noticeably, there was no significant difference in the distribution of estimated gastric acid secretion between the PDS and EPS groups, although the proportion of hypersecretors (vs non-hyper-secretors) was greater in EPS that in PDS (27.0\% vs $14.0 \%, P=0.161$ ) (Table 1 and Fig. 2).

Of the 83 patients who completed the study protocol of 4-week acotiamide treatment, 57 responded to the therapy, and the remaining 26 did not respond, resulting in an overall response rate of $68.7 \%$. In the majority of patients $(86.5 \%)$, good compliance (over $90.0 \%$ ) was confirmed. The response rates were $66.0 \%$ for PDS and $73.1 \%$ for EPS, with no significant difference in the response rate being noted between the groups (Fig. 3). In addition, both the PDS and EPS symptom severity scores were significantly decreased by treatment in patients with PDF and EPS (from 7.0 [2.0]

Table 1. Comparisons of Clinical Features According to the Type of Functional Dyspepsia

\begin{tabular}{|c|c|c|c|c|c|}
\hline Type of FD & Total $(\mathrm{N}=86)$ & $\operatorname{PDS}(\mathrm{n}=56)$ & $\operatorname{EPS}(n=26)$ & Overlap $(n=4)$ & $\begin{array}{c}P \text {-value } \\
(\mathrm{PDS} \text { vs EPS) }\end{array}$ \\
\hline Age (yr) & $54.8(15.2)$ & $54.2(16.0)$ & $56.8(13.4)$ & $50.0(16.6)$ & 0.481 \\
\hline Age ( $\geq 60 \mathrm{yr})$ & $27 / 86(31.4 \%)$ & $16 / 56(28.5 \%)$ & $10 / 26(38.5 \%)$ & $1 / 4(25.0 \%)$ & 0.371 \\
\hline Gender (male) & $24 / 86(27.9 \%)$ & $13 / 56(23.2 \%)$ & $8 / 26(30.8 \%)$ & $3 / 4(75.0 \%)$ & 0.473 \\
\hline $\operatorname{BMI}\left(\geq 23.0 \mathrm{~kg} / \mathrm{m}^{2}\right)$ & $27 / 86(31.4 \%)$ & $15 / 56(26.8 \%)$ & $9 / 26(34.6 \%)$ & $3 / 4(75.0 \%)$ & 0.475 \\
\hline Current smoker & $10 / 86(11.6 \%)$ & $4 / 56(7.1 \%)$ & $4 / 26(15.4 \%)$ & $2 / 4(50.0 \%)$ & 0.240 \\
\hline Current drinker & $49 / 86(57.0 \%)$ & $30 / 56(53.6 \%)$ & $16 / 26(61.5 \%)$ & $3 / 4(75.0 \%)$ & 0.505 \\
\hline $\begin{array}{l}\text { Illness period } \\
(\geq 1 \mathrm{mo} \\
<3 \mathrm{mo} / \geq 3 \mathrm{mo} \\
<6 \mathrm{mo} / \geq 6 \mathrm{mo})\end{array}$ & $\begin{array}{c}52 / 14 / 20 \\
(60.5 \% / 16.3 \% / 23.2 \%)\end{array}$ & $\begin{array}{c}35 / 8 / 13 \\
(62.5 \% / 14.3 \% / 23.3 \%)\end{array}$ & $\begin{array}{c}15 / 5 / 6 \\
(57.7 \% / 19.2 \% / 23.1 \%)\end{array}$ & $\begin{array}{c}2 / 1 / 1 \\
(50.0 \% / 25.0 \% / 25.0 \%)\end{array}$ & 0.848 \\
\hline sPG I (ng/mL) & $47.6(55.9)$ & $45.5(33.0)$ & $54.9(89.9)$ & $29.5(11.2)$ & 0.491 \\
\hline sPG II (ng/mL) & $10.6(10.3)$ & $10.1(7.7)$ & $11.7(14.9)$ & $10.1(7.6)$ & 0.516 \\
\hline sPG I/II ratio & $4.6(1.5)$ & $4.8(1.5)$ & $4.4(1.3)$ & $4.1(2.3)$ & 0.262 \\
\hline H. pylori (neg./pos./era.) & $\begin{array}{c}62 / 11 / 13 \\
(72.1 \% / 12.8 \% / 15.1 \%)\end{array}$ & $\begin{array}{c}42 / 7 / 7 \\
(75.0 \% / 12.5 \% / 12.5 \%)\end{array}$ & $\begin{array}{c}18 / 5 / 3 \\
(69.3 \% / 19.2 \% / 11.5 \%)\end{array}$ & $\begin{array}{c}2 / 1 / 1 \\
(50.0 \% / 25.0 \% / 25.0 \%)\end{array}$ & 0.727 \\
\hline $\begin{array}{l}\text { Estimated gastric } \\
\text { acid secretion } \\
\text { (hypo/norm/hyper) }\end{array}$ & $\begin{array}{c}44 / 27 / 15 \\
(51.2 \% / 31.4 \% / 17.4 \%)\end{array}$ & $\begin{array}{c}27 / 21 / 8 \\
(48.2 \% / 37.5 \% / 14.3 \%)\end{array}$ & $\begin{array}{c}14 / 5 / 7 \\
(53.8 \% / 19.3 \% / 26.9 \%)\end{array}$ & $\begin{array}{c}3 / 1 / 0 \\
(75.0 \% / 25.0 \% / 0.0 \%)\end{array}$ & 0.353 \\
\hline
\end{tabular}

FD, functional dyspepsia; PDS, postprandial distress syndrome; EPS, epigastric pain syndrome; BMI, body mass index; sPG, serum pepsinogen; H. pylori, Helicobacter pylori; neg., negative; pos., positive; era., eradicated.

Values are presented as mean (SD) or $\mathrm{n}(\%)$. 
A

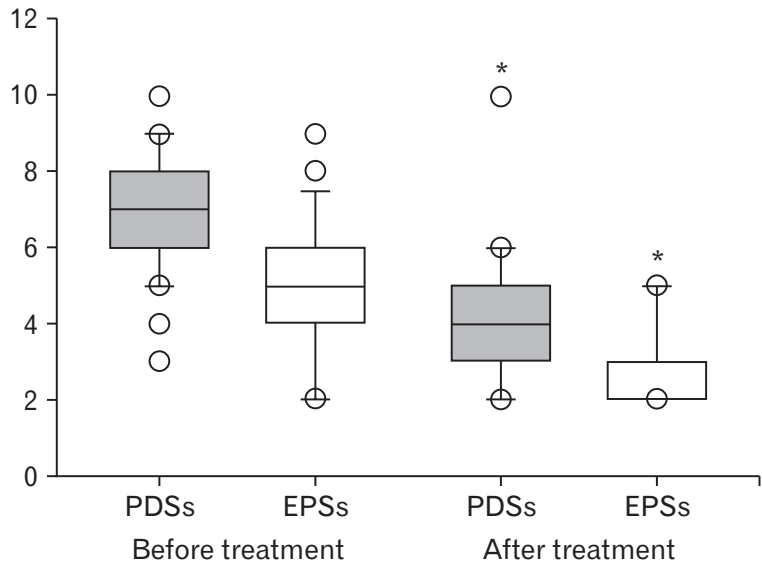

B

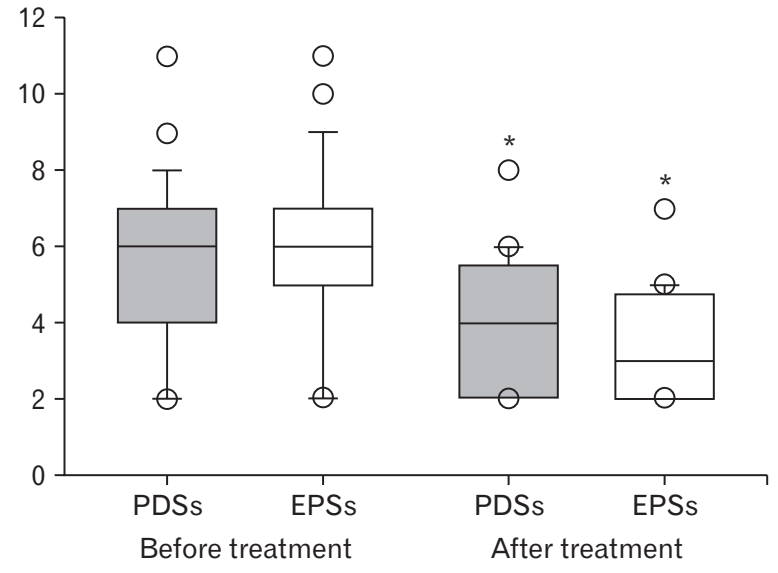

Figure 1. Box-and-whisker plots representing changes in postprandial distress syndrome (PDS) and epigastric pain syndrome (EPS) symptom severity scores (PDSs and EPSs) prior to and after 4-week treatment with acotiamide in patients with PDS (A) and patients with EPS (B). ${ }^{*}<<$ 0.01 compared with the pre-treatment value.
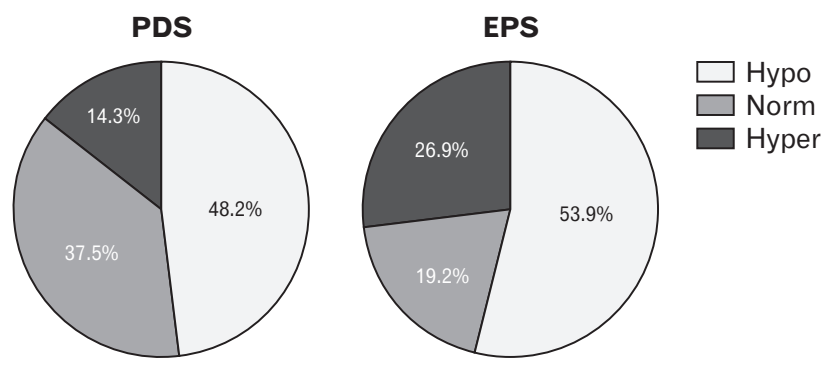

Figure 2. Distribution of estimated gastric acid secretion levels in postprandial distress syndrome (PDS) and epigastric pain syndrome (EPS). Individual gastric acid secretion levels were estimated by a combination of serum pepsinogen values and the Helicobacter pylori infection status. There were no significant differences in levels between PDS and EPS.

to $4.0[2.0]$ and from 5.0 [2.0] to 3.0 [1.0] for the PDS and EPS scores in patients with PDS, respectively, and from 6.0 [3.0] to 4.0 [3.0] and from 6.0 [2.0] to 3.0 [2.5] for the PDS and EPS scores in patients with EPS, respectively; $P<0.01$ for all comparisons) (Fig. 1). On comparing the clinical parameters between responders and non-responders, no parameters were significantly different between the 2 groups (Table 2).

Of note, the distribution of estimated gastric acid secretion in responders was hypo-secretors in $56.1 \%$, normo-secretors in $28.1 \%$, and hyper-secretors in $15.8 \%$, showing no significant difference from non-responders (hypo-secretors: $46.1 \%$, normosecretors: $30.8 \%$, and hyper-secretors: $23.1 \%, P=0.639$ ) (Table 2). However, a slightly different distribution in the estimated gastric acid secretion in relation to responsiveness to the treatment emerged in the subgroup analysis by PDS or EPS, separately. In PDS, the

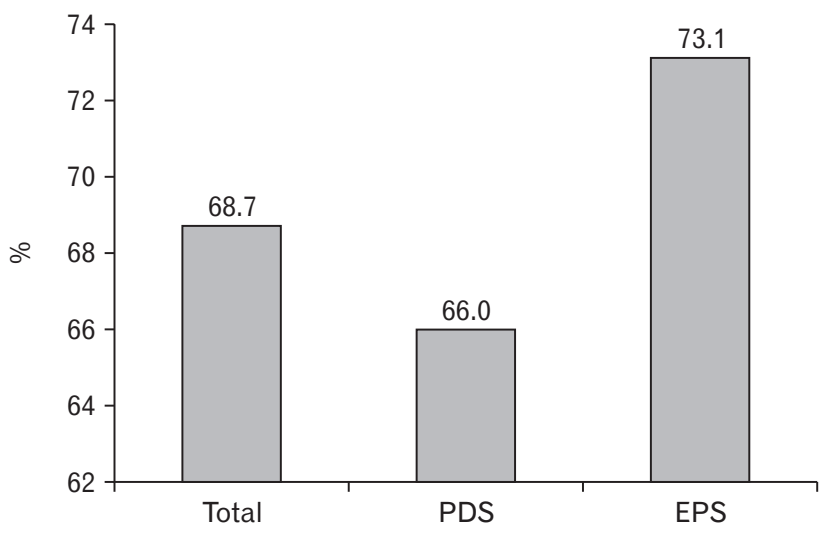

Figure 3. Response rates to acotiamide treatment in the total cohort and for postprandial distress syndrome (PDS) and epigastric pain syndrome (EPS) separately.

response rates were stable, ranging from $61.0 \%$ to $75.0 \%$, regardless of the estimated gastric acid secretion level. In contrast, in EPS, although the response rate was high in hypo-secretors (85.0\%) and normo-secretors (80.0\%), it was markedly lower in hyper-secretors (42.0\%). Thus, although the response rate did not differ notably in any acid secretion group among patients with PDS, it was significantly lower in hyper-secretors than in non-hyper-secretors among patients with $\mathrm{EPS}(P=0.046)$ (Fig. 4).

\section{Discussion}

In this study, using the combination of the $H$. pylori infection status and sPG as reliable parameters to predict the gastric acid secretion level, we found that the gastric acid secretion level did 
Table 2. Comparisons of Clinical Parameters Between Responders and Non-responders to Acotiamide Treatment

\begin{tabular}{|c|c|c|c|}
\hline Responsiveness & Responder $(\mathrm{n}=57)$ & Non-responder $(\mathrm{n}=26)$ & $P$-value \\
\hline Age (yr) & $52.7(14.3)$ & $58.3(16.7)$ & 0.120 \\
\hline $\operatorname{Age}(\geq 60 \mathrm{yr})$ & $15 / 57(26.3 \%)$ & $11 / 26(42.3 \%)$ & 0.156 \\
\hline Gender (men) & $18 / 57(31.6 \%)$ & $8 / 26(23.1 \%)$ & 0.432 \\
\hline $\operatorname{BMI}\left(\geq 23.0 \mathrm{~kg} / \mathrm{m}^{2}\right)$ & $19 / 57(33.3 \%)$ & $7 / 26(26.9 \%)$ & 0.344 \\
\hline Current smoker & $7 / 57(12.3 \%)$ & $3 / 26(15.3 \%)$ & 0.927 \\
\hline Current drinker & $36 / 57(63.1 \%)$ & $13 / 26(50.0 \%)$ & 0.268 \\
\hline Illness period $(\geq 1 \mathrm{mo},<3 \mathrm{mo} / \geq 3 \mathrm{mo},<6 \mathrm{mo} / \geq 6 \mathrm{mo})$ & $34 / 9 / 14(59.6 \% / 15.8 \% / 24.6 \%)$ & $18 / 4 / 4(69.2 \% / 15.4 \% / 15.4 \%)$ & 0.961 \\
\hline Type of FD (PDS/EPS/overlap) & $35 / 19 / 3(61.4 \% / 33.3 \% / 5.3 \%)$ & $18 / 7 / 1(69.2 \% / 26.9 \% / 3.9 \%)$ & 0.402 \\
\hline Compliance $(\geq 90 \%)$ & $48 / 57(84.2 \%)$ & $23 / 26(88.5 \%)$ & 0.616 \\
\hline sPG I (ng/mL) & $42.3(31.8)$ & $59.2(90.3)$ & 0.217 \\
\hline sPG II (ng/mL) & $9.7(7.3)$ & $12.0(15.2)$ & 0.363 \\
\hline sPG I/II ratio & $4.6(1.6)$ & $4.9(1.4)$ & 0.384 \\
\hline H. pylori (neg./pos./era.) & $45 / 6 / 6(59.0 \% / 10.5 \% / 10.5 \%)$ & $16 / 4 / 6(61.5 \% / 15.4 \% / 23.1 \%)$ & 0.226 \\
\hline Estimated gastric acid secretion (hypo/norm/hyper) & $32 / 16 / 9(56.1 \% / 28.1 \% / 15.8 \%)$ & $12 / 8 / 6(46.1 \% / 30.8 \% / 23.1 \%)$ & 0.639 \\
\hline
\end{tabular}

BMI, body mass index; FD, functional dyspepsia; PDS, postprandial distress syndrome; EPS, epigastric pain syndrome; sPG, serum pepsinogen; H. pylori, Helicobacter pylori; neg., negative; pos., positive; era., eradicated.

Values are presented as mean (SD) or $\mathrm{n}(\%)$.

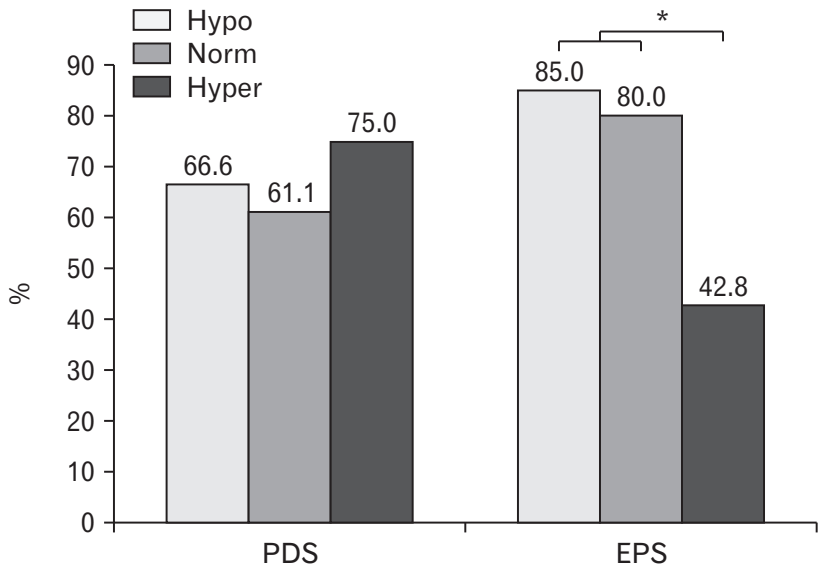

Figure 4. Response rates to acotiamide treatment in postprandial distress syndrome (PDS) and epigastric pain syndrome (EPS) according to the estimated gastric acid secretion levels. Individual gastric acid secretion levels were estimated by a combination of serum pepsinogen values and the Helicobacter pylori infection status. In EPS, the response rate was significantly lower in gastric hyper-secretors than in non-hyper-secretors. ${ }^{*} P<0.05$.

not markedly affect the phenotype of FD or responsiveness to acotiamide. Nonetheless, although treatment with acotiamide showed relatively high efficacy not only against PDS but also EPS, we found several differences in the therapeutic responsiveness between the 2 subtypes of $\mathrm{FD}$, depending on the gastric acid secretion level. For example, while treatment with acotiamide was effective among PDS cases, irrespective of the gastric acid secretion level, the efficacy may be limited in cases of EPS among hyper-secretors of gastric acid.

Since the sPG values correlate with the gastric physiological function, the measurement of the parameters (I, II, or I/II ratio) has been used to predict the relative risk of various upper gastrointestinal diseases. ${ }^{22}$ There have also been several attempts to connect these parameters to clinical aspects of $\mathrm{FD}$ in order to differentiate subtypes of the disease and predict the therapeutic responsiveness to anti-secretory drugs, although the results have been conflicting. ${ }^{27,28}$ In this multi-institutional prospective study with primary care clinics, by employing a reliable cutoff sPG value to predict the gastric acid secretion level, we investigated the influence of the estimated gastric acid secretion level on differentiating PDS and EPS and predicting the therapeutic responsiveness to the new prokinetic drug acotiamide.

FD is divided into 2 major symptomatic phenotypes-PDS and EPS - based on the assumption that different pathophysiological mechanisms underlie different dyspeptic symptoms. However, while a few studies successfully demonstrated significant differences in the underlying pathogenesis between PDS and EPS, 3,4 most other studies failed to show any marked difference in the pathophysiological mechanisms, such as gastric hypersensitivity, impaired gastric accommodation, and delayed gastric emptying. ${ }^{5-7}$ Likewise, although gastric acid is considered another important factor involved in the pathogenesis of $\mathrm{FD}$, the current study failed to show a significantly different distribution of estimated gastric secretion between PDS and EPS. This may be consistent with previous reports that the gastric acid secretion in cases of non-ulcer dyspep- 
sia is similar to that in healthy controls, irrespective of the type of symptoms, although those studies were performed before the era in which FD was subdivided into the current classification (eg, PDS and EPS). ${ }^{29,30}$

Since a phase II trial showed that acotiamide has beneficial effects against FD, particularly PDS, ${ }^{31}$ a phase III trial was conducted in PDS alone, and successfully demonstrated beneficial effects in such subgroup of FD. ${ }^{12}$ However, the phase II study also demonstrated some beneficial effects on EPS symptoms, such as upper abdominal pain. ${ }^{31}$ Consistent with this, a recent single-center retrospective study reported a similarly high efficacy of acotiamide not only against PDS but also EPS (78.0\% vs 70.0\%). ${ }^{13}$ We reinforced this finding through our multi-center prospective study and found a high drug efficacy for PDS as well as EPS (66.0\% vs $73.1 \%$ ). Such a broad effect of acotiamide may contrast with those of other prokinetic drugs, like itopride, which is effective against PDS alone. ${ }^{32}$ Previous studies on the pharmacological action of acotiamide in human and animal models demonstrated that, in addition to local effects on gut motility, acotiamide may yield beneficial effects on dyspeptic symptoms indirectly through the brain-gut axis via the effects of ghrelin on the central nervous system. ${ }^{10}$ Such diverse pharmacological effects of acotiamide may explain the broad responsiveness in $\mathrm{FD}$.

Two retrospective single-center studies reported clinical factors for predicting the responsiveness of acotiamide in FD treatment, showing conflicting results. ${ }^{13,14}$ Overall, the current study failed to show any significant difference in any clinical parameters, including the type of FD and estimated gastric acid secretion. Nonetheless, when examining the association of the estimated gastric acid secretion with the therapeutic responsiveness of acotiamide in PDS and EPS separately, we found different response profiles between the 2 types of FD. While the efficacy of acotiamide against PDS seemed to be independent of the gastric acid secretion level, the efficacy against EPS was significantly lower in gastric hyper-secretors than in non-hyper-secretors, suggesting that the efficacy of acotiamide in EPS may be affected by the secretion level. Thus, although acotiamide seems promising for the treatment of EPS as well as PDS overall, its efficacy for EPS may be limited in hyper-secretors, for whom acid suppression with a PPI is a reasonable alternative therapeutic option. Therefore, estimating the gastric secretion level by sPG and the $H$. pylori status may be useful for selecting the firstline therapeutic approach for patients with EPS.

Our current finding of insufficient efficacy of acotiamide in EPS with gastric hyper-secretion is consistent with a previous finding from an animal model. In that study, while pentagastrin- induced gastric acid hyper-secretion aggravated the pain response to gastric dilation by delaying gastric emptying, the histamine $\mathrm{H} 2$ blocker famotidine, but not the prokinetic drug mosapride, successfully ameliorated the pain response. ${ }^{33}$

Several limitations associated with the present study warrant mention. First, regarding the illness period, we defined dyspeptic symptoms lasting for more than 1 month as FD. Since the guideline of the Japanese Society of Gastroenterology defines just "chronic symptoms" of dyspepsia as $\mathrm{FD},{ }^{1}$ in many previous studies conducted in Japan, FD was diagnosed with the same criteria as the current study. ${ }^{13,14,34}$ However, according to the international consensus guideline (Rome IV), persistent dyspeptic symptoms for at least 3 months are required to diagnose FD. ${ }^{2}$ Because of easy access to medical care through the national health insurance system in Japan, only a few Japanese patients wait for more than 3 months before consulting doctors, ${ }^{1,34,35}$ which held true in the current study subjects. Nonetheless, a previous study demonstrated that the quality of life impairment by dyspeptic symptoms was similar between patients with an illness period of $\geq 1$ month and those with a period of $\geq 6$ months in Japanese patients with $\mathrm{FD}^{34}$ implying the clinical relevance of dyspeptic symptoms irrespective of the duration of the disorders. In the current study, the illness periods were evenly distributed with regard to the type of symptoms or drug responsiveness (Tables 1 and 2); we therefore believe that the illness period had little effect on the current outcomes. Second, the number of enrolled patients was relatively small. We initially planned a sample size of 200 considering feasibility, but we successfully enrolled only 86 patients. The small number of patients precluded a multivariate analysis for determining the clinical parameters predictive of therapeutic responsiveness. In addition, since we found a trend toward an association between the type of FD and estimated gastric acid secretion, a type II error due to an insufficient number of subjects may have been responsible for the insignificant association. Third, subgrouping of the symptoms (eg, PDS, EPS, or overlapping) was performed based on each patient's chief complaint, not by the symptom score, although the classification method of the subtype of FD symptoms was not consistent in previous studies. ${ }^{3-7}$ Then, the efficacy of the treatment was also evaluated by an OTE probably on the chief complaints, not by the individual symptom score. The advantage of subgrouping based on each patient's chief complaint is that it closely resembles the way in which doctors see patients with a specific chief complaint in the clinical setting. Accordingly, the patients were classified into either PDS or EPS based on their chief complaint as much as possible in the current study; this minimized the proportion of overlapping to $4.6 \%$, which is much 
lower than the value in previous studies using symptom scores to classify subgroup (16.0-25.0\% $)^{36,37}$ where PDS- and EPS-related symptoms are independently evaluated by the score in each patient. Furthermore, since our patients with EPS had similarly high PDS and EPS symptom severity scores, they may have included some EPS plus overlapping patients in addition to "pure EPS" patients, which may be partly responsible for the high response rate for EPS in the current study. Finally, regarding the study design, the lack of a placebo arm is another limitation of this study, as it is well recognized that placebo effects are not negligible, especially in the treatment of FD. However, a previous randomized control trial already demonstrated the significant efficacy of acotiamide over placebo, ${ }^{12}$ and we wished to focus on investigating predictive factors for the therapeutic responsiveness among drug users. Similarly, the lack of an observation period (eg, 1 or 2 weeks) between registration and treatment commencement may have been responsible for the overall high response rate in the current study, as FD symptoms may have disappeared spontaneously during the observation period.

In conclusion, although the prokinetic drug acotiamide is effective for treating EPS as well as PDS, the efficacy is somewhat limited in EPS patients with gastric acid hyper-secretion, in whom gastric acid inhibitors such as PPI would be more suitable. Estimation of the gastric acid secretion level by a combination of sPG and the $H$. pylori status may be useful for selecting the appropriate firstline treatment for FD, especially EPS.

\section{Financial support: None.}

\section{Conflicts of interest: None.}

Author contributions: Toshiaki Suzuki collected and analyzed the data, and drafted the article; Reina Ohba and Yosuke Shimodaira analyzed the data; Ei Kataoka, Yui Kudo, Akira Zeniya, Daisuke Segawa, Keisuke Oikawa, Masaru Odashima, Taiji Saga, Tomoyuki Kuramitsu, Hideaki Sasahara, Kazuo Yoneyama, and Takashi Tomita analyzed the data; and Katsunori Iijima designed the study, collected and analyzed the data, and drafted the article.

\section{References}

1. Miwa H, Kusano M, Arisawa T, et al. Evidence-based clinical practice guidelines for functional dyspepsia. J Gastroenterol 2015;50:125-139.

2. Stanghellini V, Chan FK, Hasler WL, et al. Gastroduodenal disorders. Gastroenterology 2016;150:1380-1392.

3. Di Stefano M, Miceli E, Tana P, et al. Fasting and postprandial gastric sensorimotor activity in functional dyspepsia: postprandial distress vs. epigastric pain syndrome. Am J Gastroenterol 2014;109:1631-1639.
4. Shindo T, Futagami S, Hiratsuka T, et al. Comparison of gastric emptying and plasma ghrelin levels in patients with functional dyspepsia and non-erosive reflux disease. Digestion 2009;79:65-72.

5. Haag S, Senf W, Tagay S, et al. Is there any association between disturbed gastrointestinal visceromotor and sensory function and impaired quality of life in functional dyspepsia? Neurogastroenterol Motil 2010;22:262e79.

6. Ochi M, Tominaga K, Tanaka F, et al. Clinical classification of subgroups according to the Rome III criteria cannot be used to distinguish the associated respective pathophysiology in Japanese patients with functional dyspepsia. Intern Med 2013;52:1289-1293.

7. Vanheel H, Carbone F, Valvekens L, et al. Pathophysiological abnormalities in functional dyspepsia subgroups according to the Rome III criteria. Am J Gastroenterol 2017;112:132-140.

8. Geeraerts B, Tack J. Functional dyspepsia: past, present, and future. J Gastroenterol 2008;43:251-255.

9. Camilleri M, Stanghellini V. Current management strategies and emerging treatments for functional dyspepsia. Nat Rev Gastroenterol Hepatol 2013;10:187-194.

10. Yamawaki H, Futagami S, Wakabayashi M, et al. Management of functional dyspepsia: state of the art and emerging therapies. Ther Adv Chronic Dis 2018;9:23-32.

11. Hsu YC, Liou JM, Yang TH, et al. Proton pump inhibitor versus prokinetic therapy in patients with functional dyspepsia: is therapeutic response predicted by Rome III subgroups? J Gastroenterol 2011;46:183-190.

12. Matsueda K, Hongo M, Tack J, Saito Y, Kato H. A placebo-controlled trial of acotiamide for meal-related symptoms of functional dyspepsia. Gut 2012;61:821-828.

13. Shinozaki S, Osawa H, Sakamoto H, Hayashi Y, Kawarai Lefor A, Yamamoto $\mathrm{H}$. The effect of acotiamide on epigastric pain syndrome and postprandial distress syndrome in patients with functional dyspepsia. J Med Invest 2016;63:230-235.

14. Miki A, Yamamoto T, Maruyama K, et al. Extensive gastric mucosal atrophy is a possible predictor of clinical effectiveness of acotiamide in patients with functional dyspepsia. Int J Clin Pharmacol Ther 2017;55:901904.

15. Miwa H, Nakajima K, Yamaguchi K, et al. Generation of dyspeptic symptoms by direct acid infusion into the stomach of healthy Japanese subjects. Aliment Pharmacol Ther 2007;26:257-264.

16. Lee KJ, Vos R, Janssens J, Tack J. Influence of duodenal acidification on the sensorimotor function of the proximal stomach in humans. Am J Physiol Gastrointest Liver Physiol 2004;286:G278-G284.

17. Iwai W, Abe Y, Iijima K, et al. Gastric hypochlorhydria is associated with an exacerbation of dyspeptic symptoms in female patients. J Gastroenterol 2013;48:214-221.

18. Iijima K, Abe Y, Kikuchi R, et al. Serum biomarker tests are useful in delineating between patients with gastric atrophy and normal, healthy stomach. World J Gastroenterol 2009;15:853-859.

19. Iijima K, Sekine H, Koike T, Imatani A, Ohara S, Shimosegawa T. Serum pepsinogen concentrations as a measure of gastric acid secretion in Helicobacter pylori-negative and -positive Japanese subjects. J Gastroenterol 2005;40:938-944. 
20. Feldman M, Richardson CT, Lam SK, Samloff IM. Comparison of gastric acid secretion rates and serum pepsinogen I and II concentrations in occidental and oriental duodenal ulcer patients. Gastroenterology 1998;95:630-635.

21. Iijima K, Koike T, Abe Y, Shimosegawa T. Cutoff serum pepsinogen values for predicting gastric acid secretion status. Tohoku J Exp Med 2014;232:293-300.

22. Iijima K, Koike T, Ara N, et al. Identification of a high-risk group for low-dose aspirin-induced gastropathy by measuring serum pepsinogen in H. pylori-infected subjects. J Gastroenterol 2015;50:305-312.

23. Junghard O, Lauritsen K, Talley NJ, Wiklund IK. Validation of seven graded diary cards for severity of dyspeptic symptoms in patients with non ulcer dyspepsia. Eur J Surg Suppl 1998:106-111.

24. Sakurai K, Nagahara A, Inoue K, et al. Efficacy of omeprazole, famotidine, mosapride and teprenone in patients with upper gastrointestinal symptoms: an omeprazole-controlled randomized study (J-FOCUS). BMC Gastroenterol 2012;12:42.

25. Ang D, Talley NJ, Simren M, Janssen P, Boeckxstaens G, Tack J. Endpoints used in functional dyspepsia drug therapy trials. Aliment Pharmacol Ther 2011;33:634-649.

26. Iijima K, Koike T, Abe Y, et al. Alteration of correlation between serum pepsinogen concentrations and gastric acid secretion after $H$. pylori eradication. J Gastroenterol 2009;44:819-825.

27. Yoshikawa I, Murata I, Kume K, et al. Serum pepsinogen can predict response to $\mathrm{H} 2$-receptor antagonist in patients with functional dyspepsia. Aliment Pharmacol Ther 2002;16:1805-1809.

28. Tahara T, Shibata T, Okubo M, et al. Examination of serum pepsinogen in functional dyspepsia. Hepatogastroenterology 2012;59:2516-2522.

29. Talley NJ, Phillips SF. Non-ulcer dyspepsia: potential causes and pathophysiology. Ann Intern Med 1988;108:865-879.
30. O Nyrén, Adami HO, Gustavsson S, Lindgren PG, Lööf L, Nyberg A. The "epigastric distress syndrome". A possible disease entity identified by history and endoscopy in patients with nonulcer dyspepsia. J Clin Gastroenterol 1987;9:303-309.

31. Tack J, Masclee A, Heading R, et al. A dose-ranging, placebo-controlled, pilot trial of Acotiamide in patients with functional dyspepsia. Neurogastroenterol Motil 2009;21:272-280.

32. Kamiya T, Shikano M, Kubota E, et al. A multicenter randomized trial comparing rabeprazole and itopride in patients with functional dyspepsia in Japan: the NAGOYA study. J Clin Biochem Nutr 2017;60:130-135.

33. Keto Y, Hirata T, Takemoto Y, Yamano M, Yokoyama T. Influence of gastric acid on gastric emptying and gastric distension-induced pain response in rats--effects of famotidine and mosapride. Neurogastroenterol Motil 2012;24:147-153, e88.

34. Kinoshita Y, Chiba T. Characteristics of Japanese patients with chronic gastritis and comparison with functional dyspepsia defined by Rome III criteria: based on the large-scale survey, FUTURE study. Intern Med 2011;50:2269-2276

35. Manabe N, Haruma K, Hata J, et al. Clinical characteristics of Japanese dyspeptic patients: is the Rome III classification applicable? Scand J Gastroenterol 2010;45:567-572.

36. Aziz I, Palsson OS, Törnblom H, Sperber AD, Whitehead WE, Simrén M. Epidemiology, clinical characteristics, and associations for symptombased Rome IV functional dyspepsia in adults in the USA, Canada, and the UK: a cross-sectional population-based study. Lancet Gastroenterol Hepatol 2018;3:252-262.

37. Suzuki H. The application of the Rome IV criteria to functional esophagogastroduodenal disorders in Asia. J Neurogastroenterol Motil 2017;23:325-333. 